\title{
Direct Transformation, Employing Modified Tixoinjection Process in Aluminum Alloy Al356.1 Chip Briquettes
}

\author{
João Roberto Sartori Moreno*
}

Fernando de Medeiros Diório, Brazil

\section{ISSN : 2688-836X}

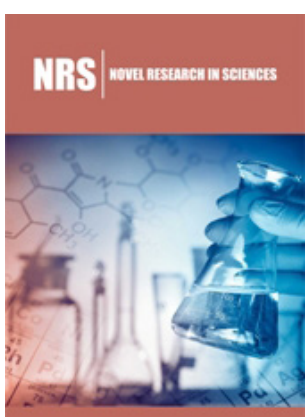

*Corresponding author: João Roberto Sartori Moreno, Fernando de Medeiros Diório, Brazil

Submission: 海June 03, 2019

Published: 柴July 11, 2019

Volume 1 - Issue 2

How to cite this article: João R S M. Direct Transformation, Employing Modified Tixoinjection Process in Aluminum Alloy Al356.1 Chip Briquettes. Nov Res Sci.1(2). NRS.000510.2019.

DOI: 10.31031/NRS.2019.1.000510

Copyright@ João Roberto Sartori Moreno, This article is distributed under the terms of the Creative Commons Attribution 4.0 International License, which permits unrestricted use and redistribution provided that the original author and source are credited.

\section{Introduction}

This work describes a proficient and innovative technique for billets production, by a modified direct thixo-injection transformation process of aluminun alloy Al356.1 chip briquettes. A thixo-injection device was built, coupled into a universal testing machine and an inductive heating system, monitored by a temperature acquisition device. The metallic solids of the globular structure, also known as refunded, are metallic alloys that have the final morphology of their microstructure changed to approximate as much as possible of a spherical geometric shape, completely escaping the traditional dendritic morphology of foundry, very common in non-alloys ferrous metals, especially in those with aluminum as the base metal. Depending on the fabrication technique employed, these refolded materials may additionally undergo a refining process, resulting in microstructures of nanometric magnitudes [1-5].

The processes for obtaining structures from liquids involve their agitation during solidification under conditions such that vigorous shear streams are produced between the layers of liquid and therefore strong friction occurs between the liquid and the solid information.

Semi-solid-state processing is capable of producing complex geometry parts without major changes in conventional manufacturing models [5]. Therefore, in this work, an unprecedented methodology for direct transformation of chips (TDC) in cylindrical billets with microstructural morphology preferentially globular-obtained from aluminum briquettes of the A356.1 aluminum alloy, followed by physical and morphological characterization.

The chemical compositions of the Al356.1 lingots were obtained and presented in the Table 1.

Table 1: Chemical composition Average of samples (\%wt).

\begin{tabular}{|c|c|c|c|c|c|c|c|c|}
\hline Material & Si & Fe & Cu & Mn & Mg & Zn & Ti & Al \\
\hline Al 356.1 & 6.59 & 0.126 & 0.19 & 0.089 & 0.334 & 0.078 & 0.198 & Bal. \\
\hline
\end{tabular}

\section{Morphological and Microstructural Analysis}

Figure 1 shows the general appearance of the microstructure, for each experimental condition evaluated, where one of the most important points in this work is the morphological and microstructural characterization. A common observation of all experimental conditions is the visible refinement of the structure, regardless of its morphology, as compared to cast structures of the same alloy. In addition, all the analyzed samples presented total transformation of the original microstructure of the chip (severely hardening) to dendritic, rosette or globular. The globularized microstructure seems to maintain a stability pattern, both dimensional and morphological, not undergoing major changes in function of the sampling site (Figure 1).

With the obtained results, it is possible to observe that, for the experimental condition of constituted globular structure (condition 6), there is a discrete variation in the values of area of the primary phase and circularity of the globules corrected by the aspect ratio, as a function of the position of measurement. Similar behavior was observed in another experimental 
condition forming a globular structure, suggesting to be a behavior independent of the influence of the factors of sample generation. On the other hand, the samples of condition 2, preferential rosette format, showed a growing increase in the area of microconstituents of the primary phase (15\%), from the base to the top, without major changes in the aspect ratio corrected circularity.
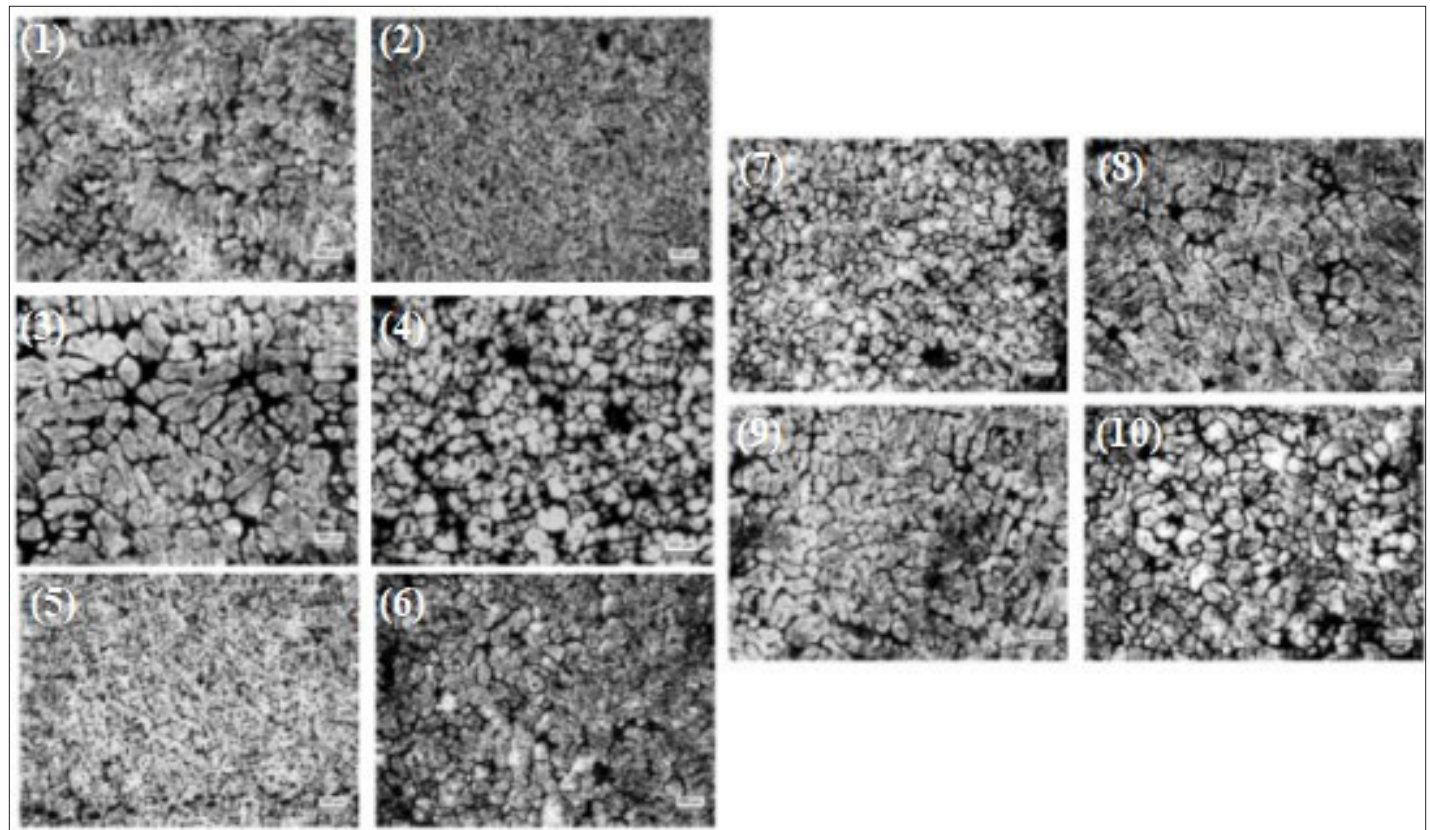

Figure 1:Microstructural morphological aspect for ingot (1) and of the 9 experimental conditions evaluated (2, $3,4,5,6,7,8,9$ and 10).

\section{References}

1. Dwivedi SP, Sharma S, Misra R (2014) Advanced materials manufacturing \& characterization A356 aluminum alloy and applications - a review. Advanced Materials Manufacturing \& Characterization.

2. Byung K, Chun PP, Byoung H, Young S, Sohn (2015) Microstructural evolution in semisolid forging of A356 alloy. Metals and Materials International 21(1): 153-158.
3. Kopanja L (2016) Quantifying shapes of nanoparticles using modified circularity and ellipticity measures. Measurement 92: 252-263.

4. LI M (2017) Secondary solidification behavior of A356 aluminum alloy prepared by the self-inoculation method. Metals 7(233): 1-18.

5. Takashimizu Y, Iiyoshi M (2016) New parameter of roundness R: circularity corrected by aspect ratio. Progress in Earth and Planetary Science 3(2). 